JOURNAL OF MODERN OPTICS, 1993, vOL. 40, NO. 1, 89-96

\title{
Double pulse-electronic speckle interferometry
}

\author{
G. PEDRINI, B. PFISTER and H. TIZIANI \\ Institut für technische Optik, Universität Stuttgart Pfaffenwaldring 9, \\ D-7000 Stuttgart 80, Germany
}

(Received 26 June 1992; revision received 7 October 1992)

\begin{abstract}
In this paper we describe a double-pulsed electronic speckle interferometry system. Two separated speckle patterns are recorded within few microseconds using a charge-coupled device camera, the two images are then stored in a frame grabber and the subtraction method is applied. A quantitative analysis of the fringes using the spatial-carrier phase-shift method is presented. The results show that this method is well suited to study transient vibrations.
\end{abstract}

\section{Introduction}

Electronic speckle pattern interferometry (ESPI) has been widely used for the deformation measurement and vibration analysis. However only when the test object is vibrating sinusoidally, it is possible to use time averaged ESPI [1-4].

For transient vibrations (e.g. in the analysis concerning the vibration produced by a running engine), we are interested to know what happens in a time interval of few microseconds. For this application it is not possible to apply time averaged techniques. Until now double-pulsed holographic interferometry has been used to measure this kind of vibration [5]. Holographic interferometry has the disadvantage that it needs the recording and the reconstruction of a hologram. For the recording a photographic plate or a thermo-plastic camera is usually used. The hologram is then reconstructed usually with a continuous laser and viewed with a charge-coupled device (CCD) camera. This process is time consuming.

Double pulsed ESPI enables one to obtain correlation fringes corresponding to the displacement without recourse to any form of photographic processing and plate relocation.

An ESPI method using a double-pulsed ruby laser is described in [6] and [7]. Where the pulse separation can be a few microseconds and the two speckle images are added using a video tube camera. The correlation fringes obtained are of poor contrast.

In this paper we will describe a double-pulsed ESPI system which uses the subtraction method. Two separated speckle patterns are recorded within few microseconds using a CCD camera. The two images are then stored in a frame grabber and the subtraction method is applied. The correlation fringes obtained are of good contrast.

The quantitative analysis of the interference fringes can be carried out in different ways. Techniques are presented and compared in [8], namely the phase shift and the Fourier methods. The Fourier method as described in [9] needs only one interferogram but is not as accurate as the phase shift method. In the phase shift method three, four or more interferograms are needed. In continuous speckle 
interferometry the shift is usually introduced with a piezoelectric transducer which can however not be used with a pulsed laser. In the spatial-carrier phase-shift methods [10] all the information necessary to reduce an interferogram to a phase map is recorded simultaneously. This method was successfully applied to the Zeiss interferometer [11] and to continuous speckle interferometry [12-15]. In this paper we will present its application to double pulsed speckle interferometry.

\section{Recording of two pulses separated by few microseconds with a CCD camera}

A laser illuminates an object with two pulses temporally separated by few microseconds. Our purpose is to record two images corresponding to the two pulses by using a CCD camera.

Consider an interline transfer CCD camera (fig. 1). This camera consists of an array of photosensors each connected to a tap on a vertical shift register. When illuminated, the photo sensors generate charges that after a period of time are transferred in the shift register which is covered to prevent generation of new charges. The time necessary to transfer the charges from the photosensors to the shift register is short ( 2 or 3 microseconds for the Sony XC-77 camera used in our experiment) since it involves only a parallel transfer from each photosensor to the adjacent one. After the charge transfer the readout of the shift register starts and the photosensors of the camera are ready to capture a new image.

For our particular case we recorded the first pulse and we transfered the charges to the shift register, after this transfer we recorded the second pulse and read the two images (first image in the shift register and second image in the photosensors) in two normal readout cycles. A triggering unit performs a RESET (transfer of the charges into the shift register) after the firing of the first pulse. To achieve this task we used an external synchronization which initiates a new readout cycle after the firing of the first laser pulse.

Before the firing of the laser pulses there should be no charges in the photosensors and in the shift register. This condition can be satisfied easily

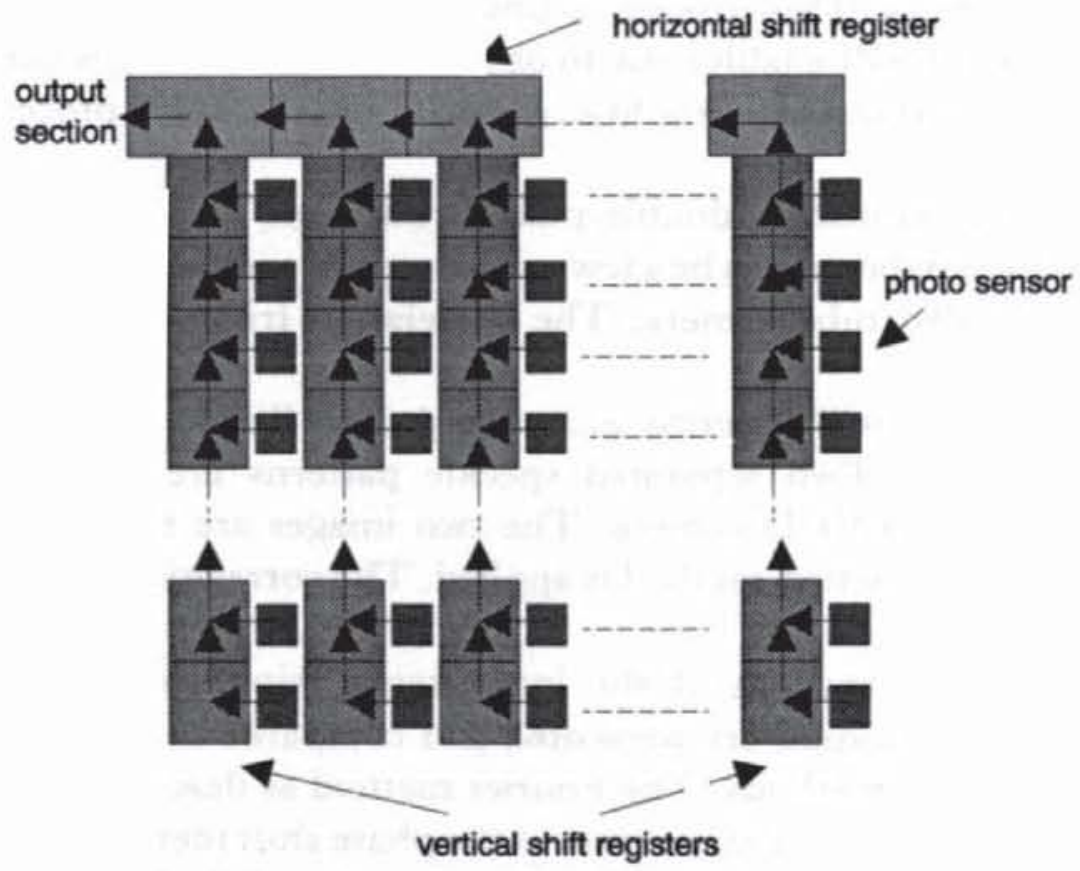

Figure 1. Interline-Transfer Imager. 
(especially when we are in a dark room), there is also no light incident on the sensors before the firing of the laser.

For our experiments we used a ruby laser (wavelength $694 \mathrm{~nm}$ ), which can emit two high energy pulses separated by few microseconds. The temporal width of each pulse is typically $20 \mathrm{~ns}$ and the temporal separation of the two pulses can be chosen to be between $1 \mu \mathrm{s}$ and $1 \mathrm{~ms}$ with an accuracy of $1 \mu \mathrm{s}$. Each pulse has typically an energy of $1 \mathrm{~J}$. The laser control unit provides an electrical pulse about $1 \mathrm{~ms}$ before the firing of the first laser pulse (this time interval can be known with an accuracy of $1 \mu \mathrm{s}$ ). This signal is used to synchronize the CCD camera. The camera used in our experiment has $756(\mathrm{H}) \times 581(\mathrm{~V})$ picture elements and each cell has a size of $11 \mu \mathrm{m} \times 11 \mu \mathrm{m}$. Since it is an interlaced camera, it is not possible to transfer the charges of all the elements at the same time but only the odd or the even lines, therefore we can use only half of the vertical resolution, but still good results can be obtained as it will be shown in the next Section. The two images are then digitalized and stored in a frame memory where they can be analysed. For our experiments we used a VFG frame grabber manufactured by Imaging Technology $(1024 \times 1024 \times 12$ bits). The stored images have the dimension of $512(\mathrm{H}) \times 256(\mathrm{~V})$ pixels and have 256 grey levels (eight bit).

\section{Electronic recording of two speckle pattern of a vibrating object}

The system used is shown in figure 2 . The beam coming from the ruby laser is split into two beams, the object beam ( $90 \%$ of the total energy) and the reference beam $\left(10 \%\right.$ of the total energy). The object beam is enlarged by a diverging lens $\left(L_{1}\right)$ and it illuminates the object $(\mathrm{O})$. The object is imaged on the CCD camera by the lens $L_{2}$ which in our experiment has a focal length of $f_{2}=60 \mathrm{~mm}$. The aperture in front of

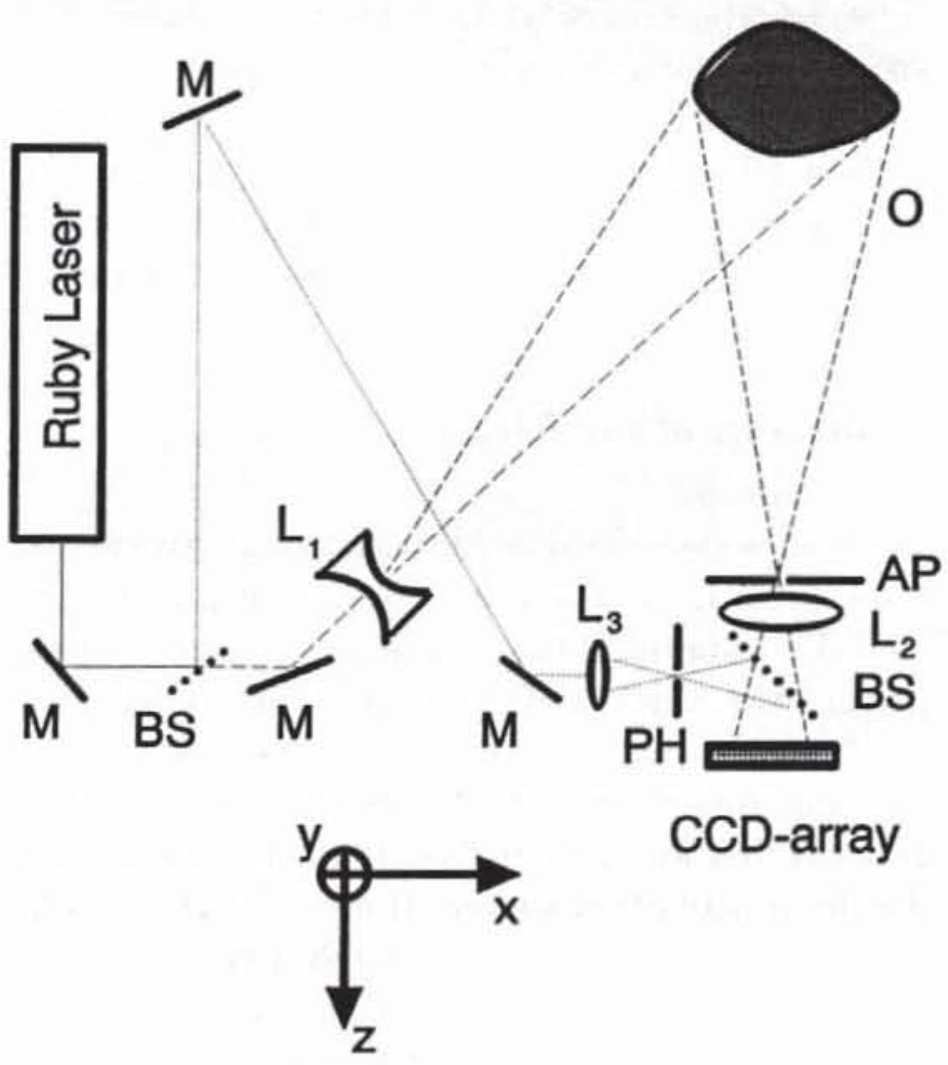

Figure 2. Experimental set-up for double pulsed ruby laser ESPI. M and BS are mirrors and beam-splitters respectively, $L_{1}, L_{2}$ and $L_{3}$ are lenses, $\mathrm{PH}$ is a pinhole, AP is an aperture. 
the lens $L_{2}$ has a diameter of about $2 \mathrm{~mm}$, the mean dimension of the speckle in the sensor plane was about $d_{\mathrm{s}}=20 \mu \mathrm{m}$. This is calculated using the well known formula $d_{\mathrm{s}}=\lambda f / D$ where $\lambda=0.69 \mu \mathrm{m}, f=60 \mathrm{~mm}$ and $D=2 \mathrm{~mm}$. The reference beam is filtered by the spatial filter composed by a microscope objective $\left(L_{3}\right)$ and a $20 \mu \mathrm{m}$ pinhole (PH). The operation of filtering is necessary because the beam coming from the ruby laser is not of good optical quality. Before the alignment of the pinhole, the reference beam and the object beam were adjusted in a manner that the virtual image of the reference point source (point where the microscope objective focalizes the beam) coincide with the centre of the aperture AP. In this manner the two beams will have about the same front-curvature. The adjustment is done by shifting the microscope objective in $x, y$ and $z$ directions. After that the pinhole is inserted. For the alignment of the system we used a He- $\mathrm{Ne}$ laser collinear with the ruby laser.

In our experiment the object used was a round metal plate $($ diameter $=200 \mathrm{~mm})$ fixed at its centre. The plate was excited using a pendulum. The two pulses were fired about $1 \mathrm{~s}$ after the impact of the pendulum with the plate. The two images of the vibrating object were recorded with a CCD camera as described in Section 2, digitalized and stored into the frame memory. Since the two laser pulses usually do not have the same energy, a normalization of the two recorded speckle images is necessary. The images are then subtracted one from the other and the absolute value is taken and stored into the frame grabber.

Figure 3 shows the results for the vibrating plate after the subtraction between the two speckle pattern. In figure $3(a)$ the pulse separation was $100 \mu$ s and in figure 3 (b) $50 \mu \mathrm{s}$. Notice that only the central part of the vibrating plate (about $18 \times 12 \mathrm{~cm}^{2}$ ) is displayed. The pictures were taken from a TV monitor. At the right side of the picture it is possible to seen the pendulum bar covered by horizontal fringes. Notice that the results shown in figure 3 represents a single mode of the plate. Given by the fact that we do not have a triggering system it was not possible to study what happens just after the shock when many modes appear to compete between themselves until one mode installs definitely. It is of course possible to record two speckle patterns separated by less than 50 microseconds. As has been seen in Section 2 that the transfer from the charges into the shift register takes only 2 or 3 microseconds. The electronics used until now did not allow to record images separated by less than $30 \mu \mathrm{s}$.

\section{Quantitative analysis of the fringes}

\subsection{Description of the method}

The Fourier method as described in [9] was used at first to analyse the fringes of figure 3 . This method needs only one interferogram but is not as accurate as the phase shift method, the obtained phase maps were of poor quality and it was not possible to reconstruct the object deformation. The spatial-carrier phase-shifting method is particularly well suited to be used for a quantitative analysis in the case of a pulsed laser. The arrangement used is practically the same as the one shown in figure 2. The only difference is that the reference beam is tilted by an angle $\theta$ with respect to the optical axis. In the image plane (where the CCD sensor is located) the speckle image of the object to be tested is then modulated with a carrier frequency having a period $p_{M}=\lambda / \sin \theta$. The angle $\theta$ is chosen so that the phase difference between the reference and object beam changes by a constant $\alpha$ from one pixel of the CCD camera to the other as shown in figure 4 . In this figure $\alpha$ was chosen to be $90^{\circ}$, thus $p_{M}=(360 / 90) \Delta x=4 \Delta x$, where $\Delta x$ is the spacing between two adjacent pixels of the 


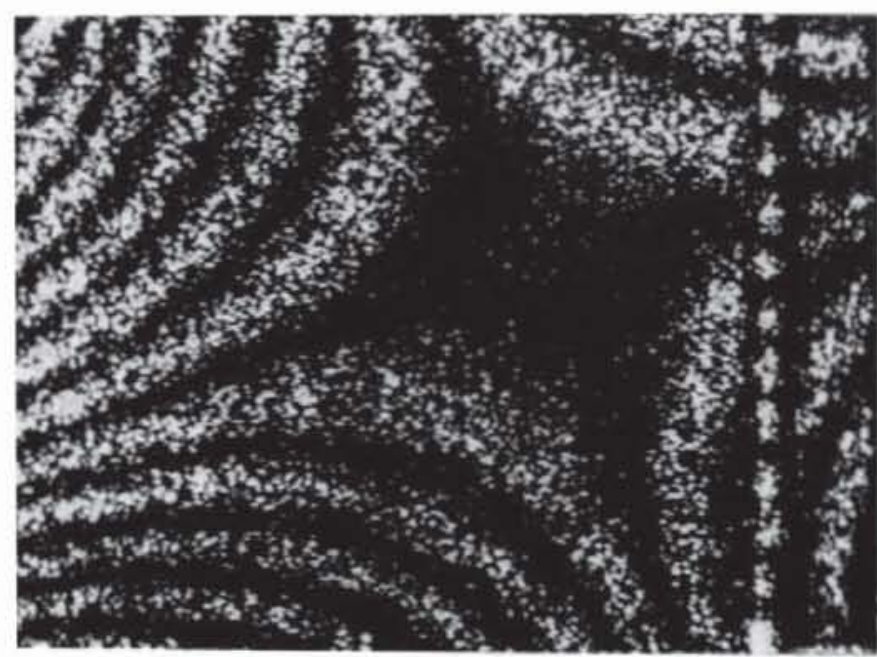

(a)

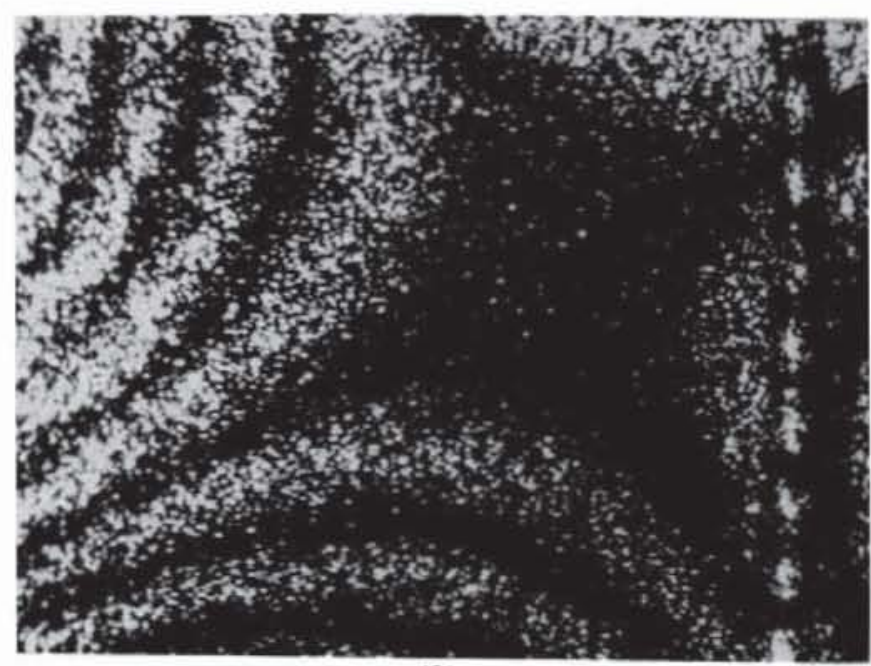

(b)

Figure 3. Speckle interferogram of a vibrating plate recorded with pulse separations of $100 \mu \mathrm{s}(a)$ and $50 \mu \mathrm{s}(b)$.

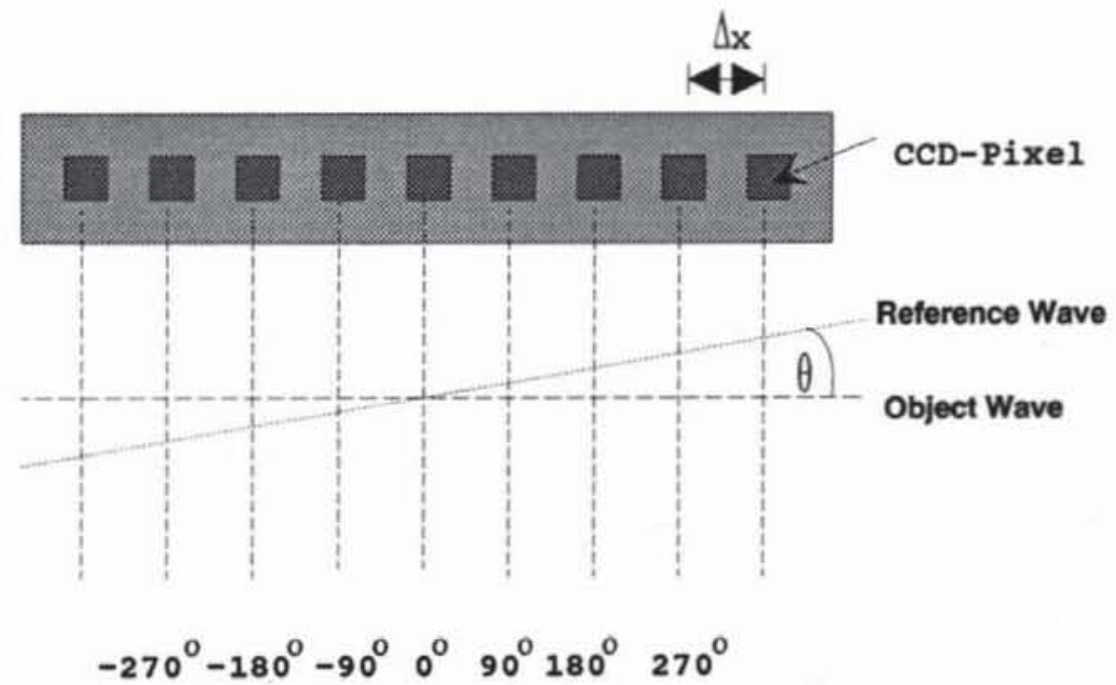

Figure 4. Relative difference between the reference and the object beam with respect to the CCD-camera pixels $\left(\alpha=90^{\circ}\right)$. 
CCD camera. The first speckle pattern $S P_{1}$ with the object in position $\mathrm{O}_{1}$ and the second $\mathrm{SP}_{2}$ with the object in position $\mathrm{O}_{2}$ are recorded and stored in the frame grabber. We considered the three-interferogram method. The intensity distribution $I_{1}$ is obtained in the following manner: the speckle pattern $S P_{2}$ is shifted one pixel left (digital shift in the frame grabber) with respect to $S P_{1}$, a subtraction between the pattern is then performed, the square module is taken and the image averaged (equation $1 a)$ )). The second interferogram with an intensity distribution $I_{2}$ is obtained by subtracting the speckle image $S P_{2}$ from $S P_{1}$, by taking the square module and by averaging (equation $(1 b)$ ). The third interferogram with intensity distribution $I_{3}$ is obtained by shifting to the right by one pixel $S P_{2}$ with respect to $S P_{1}$, by subtracting, by taking the square module again and averaging (equation $(1 c))$.

$$
\begin{aligned}
& I_{1}(x, y)=\left\langle\left|S P_{1}(x, y)-S P_{2}(x-\Delta x, y)\right|\right\rangle, \\
& I_{2}(x, y)=\left\langle\left|S P_{1}(x, y)-S P_{2}(x, y)\right|\right\rangle, \\
& I_{3}(x, y)=\left\langle\left|S P_{1}(x, y)-S P_{2}(x+\Delta x, y)\right|\right\rangle .
\end{aligned}
$$

The three intensities can thus be written in the following manner:

$$
\begin{aligned}
& I_{1}(x, y)=I_{01}(x, y)\left\{1+b_{1}(x, y) \cos [\phi(x, y)-\alpha]\right\}, \\
& I_{2}(x, y)=I_{02}(x, y)\left\{1+b_{2}(x, y) \cos [\phi(x, y)]\right\}, \\
& I_{3}(x, y)=I_{03}(x, y)\left\{1+b_{3}(x, y) \cos [\phi(x, y)+\alpha]\right\} .
\end{aligned}
$$

$I_{0 i}(i=1,2,3)$ are the average intensities, $b_{i}$ are the modulation factors, $\alpha$ is the constant phase shift and $\phi$ is the interference phase that has to be extracted. In order to apply this method it is necessary that the speckles are still correlated after the image shift of one pixel, this involves that the pixel size should be greater than the period $p_{\mathrm{M}}$. If this condition is satisfied we will obtain $I_{01} \simeq I_{02} \simeq I_{03}$, and $b_{1} \simeq b_{2} \simeq b_{3}$. In this case equation (2) become a system with three equations and three unknowns, it is thus possible to calculate the phase $\phi(x, y)$ at each point by using the well-known relation:

$$
\phi=\arctan \left(\frac{I_{3}-I_{1}}{I_{3}+I_{1}-2 I_{2}}\right) \tan \frac{\alpha}{2},
$$

where $\tan (\alpha / 2)$ is unity if the phase shift is $90^{\circ}$. For more details see [13].

\subsection{Experimental results}

The experimental set-up used in Section 3 is considered. Only two modifications were introduced to perform the experiments with the off axis reference.

(1) the beam-splitter was slightly tilted in order to have an angle $\theta$ between the reference and object beams. This angle was chosen equal to $0.69 / 44 \mathrm{rad}$, in order to have a modulation period $p_{M}=44 \mu \mathrm{m}=4 \Delta x$.

(2) the aperture was a slit $\left(1 \times 3 \mathrm{~mm}^{2}\right)$. Using such an aperture the mean speckle dimension in horizontal direction ( $x$ direction) was $d_{\mathrm{s}}=41 \mu \mathrm{m} .\left(d_{\mathrm{s}}=\lambda f / D\right.$, where $\lambda=0.69 \mu \mathrm{m}, f=60 \mathrm{~mm}$ and $D=1 \mathrm{~mm}$ ). This speckle size is about equal to $4 \times 11 \mu \mathrm{m}(4 \times$ pixel size $)$. The speckle dimension in the $y$ direction can be smaller and in our case was about $13 \mu \mathrm{m}$. 
The plate was excited using a pendulum and the two pulses were fired about $1 \mathrm{~s}$ after the impact of the pendulum with the plate, the pulse separation was $200 \mu$ s. The two images of the vibrating object were recorded with a CCD camera as described in Section 2, digitalized and stored in the frame memory. The two speckle patterns are normalized and the digital method described in $4 \cdot 1$ is applied. The three interferograms obtained are shown in figure $5(a-c)$. Notice that the image $(a)$ is about the 'inverse' of the image $(c)$, this means that the phase shift between these two images is about $180^{\circ}$. The three images are than filtered by using a $7 \times 7$ convolution low pass filter and a $7 \times 7$ convolution mean filter. After the filtering operation the interferograms were evaluated by using equation (3). The phase map is shown in figure $5(d)$. Figure $5(e)$ shows a pseudo $3 \mathrm{D}$ representation of the deformation of the central part of the plate, troubles caused by the pendulum crossing the object can be seen (the diameter of the analysed area in figure $5(e)$ was about $10 \mathrm{~cm}$ ).

(a)

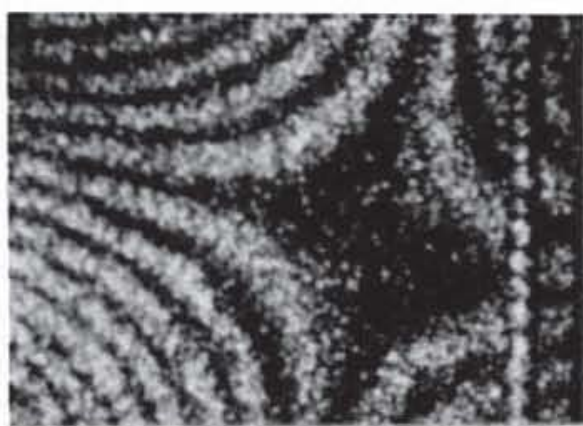

(c)

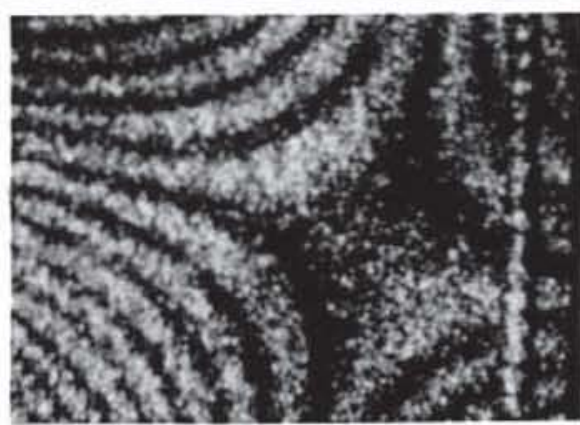

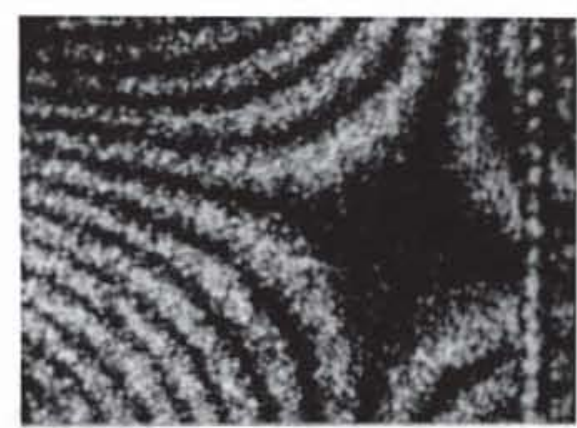

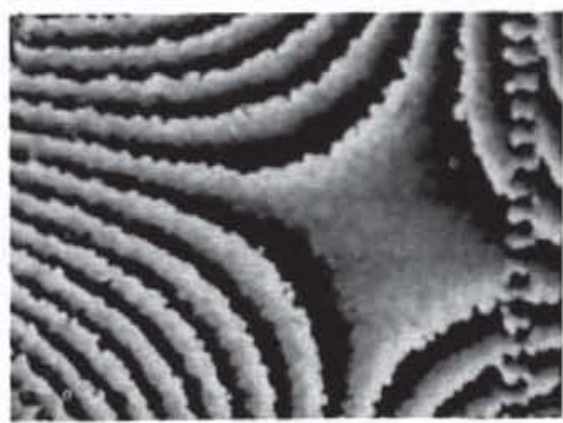

(b)

(d)

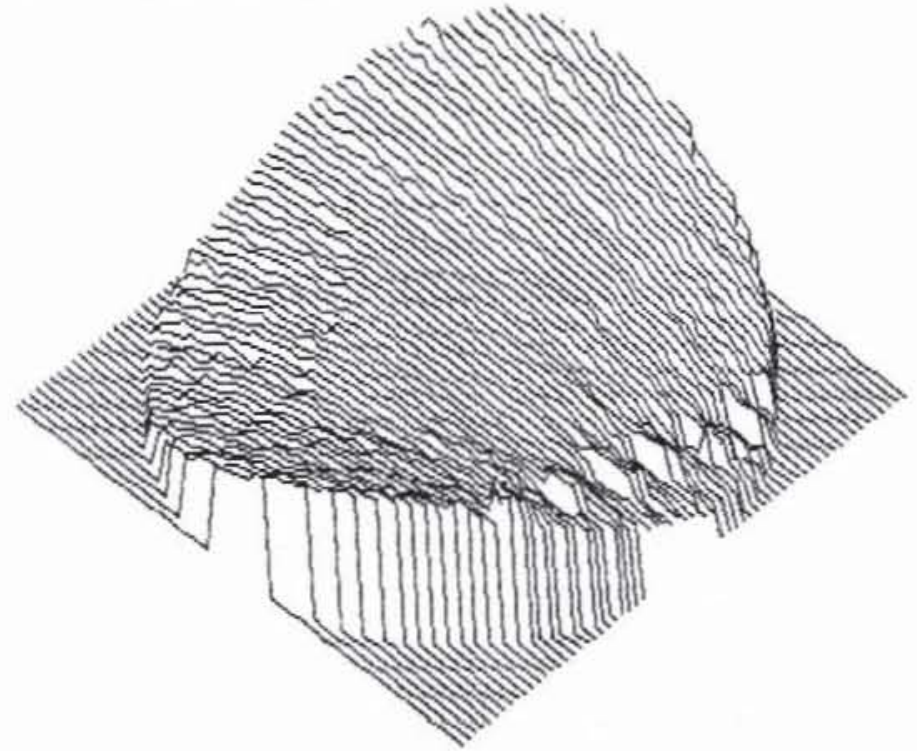

(e)

Figure 5. Phase-shifted speckle interferograms of a vibrating plate recorded with a pulse separation of $200 \mu \mathrm{s}$. (a) $-90^{\circ},(b) 0^{\circ},(c) 90^{\circ} \mathrm{C}$, (d) phase-map, (e) pseudo-3D representation of the deformation. 


\section{Conclusions}

The double-pulsed speckle interferometry method described in this paper, is a powerful tool for the analysis of vibrations. In particular the pulse separation in the range of 0.01 to $1 \mathrm{~ms}$ will allow to study transient events.

The spatial-carrier phase-shift method can be applied without problems using a two pulse ruby laser and this allows a quantitative analysis of the fringes.

This method is very much simpler than the double pulse holographic interferometry and allows a quick analysis of the interferograms without the development of films and hologram reconstructions. It is thus well suited to be used in an industrial environment.

At present the quantitative analysis is not as accurate as in the case of holographic interferometry. In fact the speckle-size during our experiments was quite large $(40 \mu \mathrm{m})$ because the CCD camera used in the experiment has pixel cell dimensions of $11 \times 11 \mu \mathrm{m}^{2}$. It is possible to use a camera with smaller pixels in order to decrease the speckle size and thus to increase the resolution.

It should be noted that during the experiments reported above, the ruby laser was operated close to its threshold (in fact the objects were quite small). By operating the ruby laser at higher energy it is possible to record interferograms of larger object e.g. $1 \times 1 \mathrm{~m}^{2}$.

\section{Acknowledgment}

The authors would like to thank Dr R. Malz, Dr X. Peng and Mr Y. L. Zou for many helpful discussions.

\section{References}

[1] Jones, R., and Wykes, C., 1989, Holographic and Speckle Interferometry (Cambridge University Press), chap. 4.

[2] Tiziani, H. J., 1978, Speckle Metrology, edited by R. K. Erf (New York: Academic), p. 73 ,

[3] Stetson, K. A., and Brohinsky, W. E., 1988, J. Opt. Soc. Am., 5, 1472.

[4] Pryputniewicz, R. J., 1989, Laser Interferometry: Quantitative Analysis of Interferograms, Proc. SPIE, 1162, 456.

[5] Sixt, W., and Engelsberger, J., 1985, Optics in Engineering Measurement, Proc. SPIE, 599, 97.

[6] Cookson, T. J., Butters, J. N., and Pollard, H. C., 1978, Optics and laser technology, $10,119$.

[7] Tyrer, J. R., 1985, Optics in Engineering Measurement. Proc. SPIE, 599, 181.

[8] Kreis, T. M., 1988, Holography Techniques and Applications, Proc. SPIE, 1026, 80.

[9] Kreis, T. M., and JüPTNer, W. P., 1989, Proc. SPIE, 1162, 116.

[10] Kujawinska, M., and WoJciak, J., 1991, Moiré Techniques, Holographic Interferometry, Optical NDT, and Applications to Fluid Mechanics, Proc. SPIE, 1554B, 503.

[11] Küchel, M., 1990, Optical Testing and Metrology III: Recent Advances in Industrial Optical Inspection, Proc. SPIE, 1332, 655.

[12] Pfister, B., Beck, M., and Tiziani, H. J., 1991, Proceedings Laser 91 (München).

[13] Pfister, B., 1992, Speckleinterferometrie zur Defektanalyse mit neuen Phasenschiebenmethoden, Dissertation Universität Stuttgart.

[14] Leidenbach, S., 1991, Proceedings Laser 91 (München).

[15] Steinbichler, H., 1991, Holographic Optics III. Principles and Applications, Proc. SPIE, 1507, 435. 